\title{
Some results and problems in dyadic analysis: an overview
}

\section{B. I. GOLUBOV}

$\begin{array}{lll}\text { Date of Receiving } & : & 01.04 .2018 \\ \text { Date of Revision } & : & 19.06 .2018 \\ \text { Date of Acceptance } & : & 08.08 .2018\end{array}$

\section{Outline}

(I) Notations.

(II) The known concepts of dyadic derivatives and integrals.

(III) Modified strong dyadic integral and derivative of fractional order on $f \in L^{2}[0,1)$.

(IV) Uniform integral in dyadic Hardy spaces.

(V) Dyadic analogue of Tauberian theorem of Wiener and related topics.

(VI) Dyadic Hardy and Hardy-Littlewood operators in the spaces $H_{d}\left(R_{+}\right)$and $B M O_{d}\left(R_{+}\right)$. (VII) Some problems.

\section{Notations}

Let us introduce some notations. For the number $x \in R_{+}=[0,+\infty)$, we will use dyadic expansion $x=\sum_{n=-\infty}^{+\infty} 2^{-n-1} x_{n}$ of $x$, where $x_{n}$ be equal to 0 or 1 . It is evident, that $x_{-n}$ for $n \geq n(x)$. If $x$ is dyadic rational, then we use finite expansion, i.e. $x_{n}=0$ for $n \geq n(x)$.

Let us introduce the generalized Walsh functions

$$
\psi(x, y) \equiv \psi_{y}(x)=(-1)^{t(x, y)},
$$

where

$$
t(x, y)=\sum_{n=-\infty}^{\infty} x_{n} y_{-n-1} \text { for }(x, y) \in R_{+} \times R_{+} .
$$

It is evident that

$$
\psi_{y}(x)=\psi_{x}(y), \psi_{y}(x)= \pm 1 \text { for } x, y \in R_{+} .
$$

Let us note that

$$
w_{n}(x) \equiv \psi_{n}(x) \equiv \psi(x, n), n \in \mathbb{Z}_{+}, x \in R_{+}
$$

2010 Mathematics Subject Classification. 42C10.

Key words and phrases. Dyadic Analysis, Walsh-Paley System, Dyadic Hardy Spaces.

Communicated by. S.S. Volosivets 